vol. 28 - $n^{\circ} 1 \mid 2012$

Migrations, transnationalisme et diaspora : théorie et études de cas

\title{
La diaspora ou l'ethnique en mouvement
}

Diaspora: A Moving Ethnicity

La diáspora o lo étnico en movimiento

Chantal Bordes-Benayoun

URL : https://journals.openedition.org/remi/5700

DOI : $10.4000 /$ remi.5700

ISSN : $1777-5418$

Éditeur

Université de Poitiers

Édition imprimée

Date de publication : 1 mars 2012

Pagination : 13-31

ISBN : 979-10-90426-03-0

ISSN : 0765-0752

Référence électronique

Chantal Bordes-Benayoun, «La diaspora ou l'ethnique en mouvement », Revue européenne des

migrations internationales [En ligne], vol. 28 - $n^{\circ} 1$ | 2012, mis en ligne le 01 mars 2015, consulté le 14

avril 2022. URL : http://journals.openedition.org/remi/5700; DOl : https://doi.org/10.4000/remi.5700 


\title{
La diaspora ou l'ethnique en mouvement
}

\author{
Chantal BORDES-BENAYOUN ${ }^{1}$
}

$D^{2}$ epuis quelques années, le terme de diaspora fait l'objet d'une floraison de travaux académiques, mais aussi d'utilisations dans le discours public. Cet engouement pour un terme, hier associé pour beaucoup, pour ne pas dire pour l'essentiel, à l'histoire des Juifs, correspond à un moment particulier dans l'évolution des phénomènes migratoires et à un changement de paradigmes dans les sciences sociales. Mal préparées, notamment en France, à saisir les mouvements identitaires des années 1980, celles-ci semblent avoir misé sur le concept de diaspora pour aborder la complexité actuelle des flux de populations. Au même moment, des peuples de plus en plus nombreux mobilisaient le terme pour défendre leurs droits (comme les revendications autochtones), organiser leur mouvement national (les Palestiniens) ou imposer l'idée de leurs intérêts communs par-delà les distances géographiques et historiques qui opposaient leurs membres (diaspora noire des Amériques). L'extrapolation à l'infini du terme à des cas aussi différents a contribué à enrichir les problématiques sur les phénomènes migratoires sans pour autant éviter la confusion pouvant naître de la profusion des discours parascientifiques. Dans l'effervescence générale, on a pu oublier la nécessité d'opérer une rupture avec le sens commun, de discerner, pour paraphraser Bachelard, entre les faits désignés par le terme et l'idée qu'il véhiculait.

Dans le contexte global, les diasporas ont pris une valeur symbolique et politique pour les peuples éparpillés désireux d'affirmer leur unité et leur présence sur la scène mondiale. Si elles évoquaient hier l'exil et l'exclusion de peuples sans attaches, elles ont aujourd'hui pris le sens positif attaché aux revendications identitaires transnationales et acquis la dimension d'un mythe (Bordes-Benayoun et Schnapper, 2006). L'utopie d'un monde sans frontières et fraternel inspire ceux qui s'y reconnaissent comme ceux qui s'y réfèrent. Par leurs vertus supposées, fidélité, solidarité, mais aussi mobilité, adaptation, inventivité, innovation, capacité à produire de la richesse, ces " championnes historiques ", douées de liberté et capables de traverser les siècles, annonceraient la venue du citoyen du monde, passant les frontières, débarrassé de ses attaches et disponible à une sorte de métissage généralisé, horizon vers lequel les sociétés devraient se diriger pour contrer les

1 Directrice de Recherches au CNRS, LISST-Centre d'anthropologie sociale-UMR 5193, Université de Toulouse le Mirail, Maison de la Recherche, 5 allées Antonio Machado, 31058 Toulouse cedex; chantal.bordes@univ-tlse2.fr 
effets négatifs de la mondialisation. Mais, en dehors de cette vision utopiste, qu'apporte vraiment de nouveau le concept dans l'analyse des mouvements humains actuels ?

\section{UNIVERSALISATION ET CHANGEMENTS DU TERME DE DIASPORA DANS L'ESPACE PUBLIC : LA DIASPORA ÉLEC- TRONIQUE}

Si l'on en juge par la récurrence du mot dans les médias et sur le web, le nombre des diasporas n'a cessé d'augmenter. On peut signaler quelques emplois métaphoriques, tels que ceux désignant la mondialisation des manuels scolaires utilisés par des chercheurs de l'Université de Laval au Québec, la communauté des mathématiciens dispersés dans le monde ou bien encore pour décrire le rassemblement de motocyclistes, etc. Emblématique est aussi le choix de ce terme pour un groupe de rap français. Tout récemment, c'est aussi ce nom qu'a adopté un réseau social concurrent de Facebook censé offrir une plus grande liberté de mouvement en s'appuyant sur des serveurs décentralisés, d'où l'image de la dispersion ${ }^{2}$. Après le décès de l'un des fondateurs du réseau, un des membres rendait compte de l'utopie sous-jacente à ce projet numérique : "This weekend we lost one of the brightest minds of the technology world, and one of our best friends. Ilya Zhitomirskiy was a crusader-or, as he called himself, a Dragonslayer-for freedom, privacy, and openness on the Internet. He believed in the power of technology to make the world a better place. And through the creation of Diaspora, he did $»^{3}$.

Mais la banalisation du terme révèle surtout qu'il tend à s'appliquer désormais soit à des phénomènes migratoires divers et d'amplitude variable, soit à des minorités ou groupes qu'un sentiment de communauté serait censé relier. Il donne à voir des « communautés virtuelles » (Proulx, Poissant et Sénécal, 2006) et tend à désigner toute forme de migration, mais aussi de communauté d'appartenance et de reconnaissance mutuelle, sans nécessaire déplacement géographique.

Sur la toile, les sites consacrés aux diasporas ont enregistré une croissance exponentielle depuis le début des années 2000 et forment un ensemble disparate, émietté et de portée inégale. Nous nous limiterons ici à quelques exemples d'initiatives en français ou en anglais dont l'intitulé comporte le terme " diaspora " ${ }^{4}$. Dans cet ensemble qui constitue un objet de recherche en soi, il s'agira simplement de saisir le sens que revêt désormais le terme dans l'espace public. L'objectif premier affiché par nombre de sites est de rétablir une continuité entre des « expatriés » et la nation ou la région d'origine. « La fréquenta-

2 Créé par quatre étudiants en informatique de New York, le nouveau réseau social " Diaspora*», ouvert en septembre 2010 s'engage auprès de ses utilisateurs à protéger la vie privée et à ne pas vendre les données personnelles (https://joindiaspora.com/).

$3 \mathrm{http}: / /$ blog.diasporafoundation.org/

4 Des sites de diaspora peuvent ne pas afficher le mot, c'est le cas de nombre de sites de diasporas classiques, comme la diaspora juive ou chinoise par exemple. On trouvera un effort bienvenu de recensement de sites web, sites universitaires, sites nationaux et sites de migrants, dans ROLLAN Françoise (2005) Une sélection de 90 sites web sur les diasporas, Cybergeo: European Journal of Geography, Espace, Société, Territoire, article 323 [en ligne]. URL : http://cybergeo.revues. org $/ 3046$ 
tion des sites diasporiques, la participation à des forums rassemblant des internautes aux mêmes origines sont appréhendées comme donnant corps à une communauté imaginée qui unit ses membres dispersés en une sorte de prolongement, dans le monde virtuel, de la nation réelle » (Mattelart, 2009 : 27). Mais la définition du territoire de référence est variable et donne d'autant plus de force à l'imagination : elle peut être régionale, nationale, continentale, transnationale, voire absente puisque, dans certains cas, la référence est totalement déterritorialisée pour être avant tout culturelle et identitaire.

Cette géographie variable tend à troubler la définition première de la diaspora comme dispersion depuis une origine donnée et à dématérialiser le centre originel au profit d'une centralité de type identitaire. Ainsi l'importance même qu'a pu jouer dans l'histoire le mythe de la terre d'origine semble moins active que l'identification à une communauté dont la base varie considérablement d'un cas à l'autre. De façon corollaire, la désignation des membres dispersés évolue d'expatriés en exilés, mais c'est le terme d' « extérieurs » qui semble l'emporter, qui met moins l'accent sur l'opposition entre étranger et national que sur la différence avec les « intérieurs ». On remarque également un émiettement en particularismes internes aux diasporas selon des critères régionaux, corporatistes, de classes ou de générations. C'est par exemple une " Diaspora Des Rugbymen Camerounais $»^{5}$ qui apparaît dans les réseaux sociaux. Plus encore, le thème de la diaspora des joueurs de football africains est largement véhiculé par les médias ${ }^{6}$.

Dans la géographie variable des diasporas d'aujourd'hui, chacun apporte une touche particulière à une entité qui se veut homogène. On parle aussi de plus en plus de diasporas scientifiques, comme pour la diaspora roumaine organisée sur le net (Nedelcu, 2009). Leur réussite rejaillit sur la communauté tout entière alors que la fuite des cerveaux est évoquée comme un exode sur le modèle des diasporas historiques ${ }^{7}$ : « Les exilés du savoir sont nombreux dans le monde et ce phénomène contribue à appauvrir davantage les pays qui sont déjà les plus pauvres. Les diasporas scientifiques représentent un espoir dans ce contexte. Une fois rassemblés pour former une nation hors du territoire d'origine, les exilés du savoir peuvent rendre de grands services à leurs compatriotes $»^{8}$.

Plusieurs sites régionaux « diasporiques » sont apparus en France dans les années 2000. Ils ont prolongé et le cas échéant recyclé les revendications régionalistes des décennies précédentes. Le site de la diaspora corse, créé en 2004, entend valoriser les liens entre les habitants de l'île et les « extérieurs ». Il revendique une diaspora forte de « 700000 à 800000 personnes, formées, dynamiques, qui ont gardé pour la plupart au fond du cœur, en dépit de l'éloignement, cet amour profond de la Corse qui ne peut que (les) unir. Dans la diaspora, chez nos amis, les exemples de réussite sociale sont

5 Association 1901, née en 2009 et réunissant une trentaine de joueurs pratiquant leur sport en Europe.

6 Le site de RFI en dresse même la liste : http://www.rfi.fr/afrique-foot/20111020-diaspora-tousfootballeurs-africains-europe-2011-2012

7 Sur la terminologie relative aux diasporas et l'opposition entre « diasporas classiques » (historiques) et « nouvelles diasporas », voir Bordes-Benayoun et Schnapper, 2008.

8 http://agora.qc.ca/francophonie.nsf/Documents/Diaspora--Diaspora_scientifique_par_Jacques_ Dufresne 
nombreux ; la multiplicité et la qualité du savoir et des compétences constituent un terreau fertile dont la Corse a impérativement besoin, aujourd'hui »'.

Cette image d'une diaspora dynamique et productive rompt avec la représentation classique associant la diaspora au malheur de l'exode et des persécutions. On retrouve cette valorisation dans de nombreux autres cas.

Plusieurs initiatives virtuelles sont consacrées à la « diaspora bretonne »dont trois ont fait l'objet d'une thèse (Le Bayon, 2010). L'intention économique est clairement affirmée par le site « diaspora-bretonne » dont la page d'accueil présente le soustitre explicite de « diaspora économique bretonne $»^{10}$. Fondé par l'Institut Locarn ${ }^{11}$, qui réunit des dirigeants économiques influents, il poursuit l'objectif d'un développement économique de la Bretagne en faisant appel aux compétences des membres éminents de la « diaspora » dont il dresse l'inventaire. D'une manière générale, on peut observer que la liste de personnalités importantes, intellectuels, artistes, entrepreneurs, politiques, vient souvent flatter la définition de la diaspora qui ressort des sites web. Un tel inventaire apparaît comme un signe des temps qui rompt avec le modèle de la discrétion adoptée par les diasporas classiques moins préoccupées de fierté ethnique que les nouvelles. La même fierté est revendiquée sur le blog de la diaspora camerounaise : « Oui je suis Camerounais et fier de l'être. C'est la raison pour laquelle je décide en commun accord avec certains compatriotes de mettre sur pied ce site afin de faire connaître et de valoriser tous ces Camerounais qui malgré l'endroit où ils se trouvent et ce qu'ils sont devenus font tout ce qui est en leur pouvoir afin de valoriser "le berceau de nos ancêtres" ${ }^{12}$.

Le site de la « diaspora nigérienne $»^{13}$, né en 2003, animé depuis Bruxelles, cultive pour sa part les sentiments patriotiques en diffusant les informations sur le pays d'origine, mais aussi sur les divers lieux d'installation de la diaspora. Il participe, selon son fondateur, à revaloriser l'image du Niger. Il faut, selon lui « étudier les leviers qui favoriseraient le retour au pays de la diaspora prête à y investir. Il serait pertinent de penser à une structure visant à promouvoir et à faciliter le retour de la diaspora et le transfert des fonds et des connaissances de l'extérieur vers l'intérieur. À titre d'exemple, il existe au Sénégal un ministère qui s'occupe de la diaspora sénégalaise et, au Mali, plusieurs services leur sont destinés. Le Niger pourrait s'inspirer de ce type d'initiatives $»^{14}$.

Cette prise de conscience du pouvoir économique de la diaspora et du poids des migrations dans les économies locales est apparue très fortement dans le cas du Sénégal, tant du côté de l'État et des acteurs économiques que du côté des établissements diasporiques. «Les Sénégalais de l'extérieur sont un levier incontournable du développement

9 www.corsicadiaspora.com/

$10 \mathrm{http}: / /$ diaspora-bretonne.com/

11 Fondé en 1991 par Joseph Le Bihan, professeur à HEC et Jean-Pierre Le Roch, créateur de l'enseigne de grande distribution « Les Mousquetaires », l'Institut de Locarn est un creuset d'idées et de projets, bénéficiant d'une ouverture sur le monde grâce à la Diaspora Économique Bretonne (http://www.institut-locarn.com/).

12 Site qui affiche son programme sous le titre : « fiers d'être camerounais - diaspora camerounaise » (http://diaspokmer.skyrock.com/).

$13 \mathrm{http}: / /$ nigerdiaspora.info/

14 www.lesahel.org

REMI 2012 (28) 1 pp. 13-31 
de notre pays. Leur poids économique est considérable et leur contribution dans l'économie nationale remarquable. D'ailleurs, l'épargne qu'ils dégagent dépasse de loin l'aide au développement de notre pays $\gg{ }^{15}$.

Dans le même sens, le Réseau International de la diaspora marocaine ${ }^{16}$, né en 2009 au Maroc, à l'initiative de cadres et dirigeants, veut « sensibiliser la diaspora à la stratégie de développement économique du Maroc et influer sur sa capacité à mobiliser des investisseurs ou à investir elle-même ; créer un réseau mondial pour mobiliser les compétences dans les pays d'accueil et constituer des groupes d'influence efficaces ».

L'image d'une diaspora dynamique et active a succédé à celle dévalorisante des migrations traditionnelles : «Ces émigrés d'un temps sont devenus une véritable communauté active dans leurs champs d'expertise dans lesquels ils sont connus et reconnus (...). [Ils forment] un véritable concentré de talents dans les domaines scientifique, culturel, politique et économique à travers le monde ${ }^{17}$.

Dépassant les spécificités des divers États, l'idée d'une diaspora africaine s'est imposée avec une égale insistance sur le potentiel représenté par les " extérieurs » dans les échanges mondiaux. La diaspora représente un enjeu économique dont États et organisations internationales se saisissent. En 2007, La Banque Mondiale met en place un programme «Diaspora africaine » qui désigne et recense « les personnes d'origine africaine vivant hors du continent africain, qui sont désireuses de contribuer à son développement et à la construction de l'Union africaine, quelles que soient leur citoyenneté et leur nationalité $»^{18}$.

Mais l'affirmation africaine ou « noire » sur le net est aussi source d'instrumentalisation politique et dans certains cas de dérives communautaristes et ethnocentristes (Fauvelle-Aymar, 2006). C'est le sens qu'entend donner l'invention d'un transnationalisme afrocentriste, sur des sites réunis autour d'une « communauté noire », dont l'histoire fait l'objet de théorisations pseudo-scientifiques fondées principalement sur une dénonciation de l'occident ${ }^{19}$. Plus proche de la notion de diaspora scientifique, et proclamant le particularisme africain, il existe une revue de mathématiques de la diaspora africaine ${ }^{20}$. Le portail d'information télé-diaspora ${ }^{21}$ conjugue pour sa part les échelles locales et transnationales, en offrant des rubriques en français et en anglais sur le Cameroun, la Côte d'Ivoire, la Guinée, le Mali et le Sénégal. C'est à l'échelle africaine et caribéenne que le site d'information « diaspora-news », créé en France récemment, se situe en s'adressant ainsi à une diaspora noire dans sa globalité22. Enfin, tout récemment le site « diasporas.

15 Le Mouvement des Entreprises du Sénégal (MEDS) par exemple, mouvement patronal fondé en 2000, a lancé un programme intitulé « diasporas des affaires » (http://www.meds-senegal.org).

$16 \mathrm{http}: / /$ www.ridm-maroc.com/nos_objectifs.php

$17 \mathrm{http}: / /$ www.ridm-maroc.com/mot_de_president.php

$18 \mathrm{http} / / /$ web.worldbank.org/WBSITE/EXTERNAL/ACCUEILEXTN/NEWSFRENCH/0,, content

MDK:22154793 pagePK:64257043 piPK:437376 theSitePK:1074931,00.html

19 Par exemple sur le site francophone : http://africamaat.com/article.php3?id_article=42

$20 \mathrm{http}: / /$ math-res-pub.org/adjm

21 www.telediaspora.net

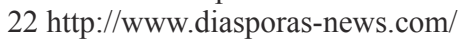


fr $»^{23}$ confirme l'affluence africaine sur le net. L'ensemble de ces initiatives éclatées, parfois minoritaires et plus ou moins éphémères n'en constituent pas moins une sorte de nébuleuse à laquelle s'accroche cahin-caha l'idée de diaspora noire africaine.

Si la dimension nationale s'efface parfois devant la progression de mouvements tournés vers l'identité africaine, elle est au contraire au centre d'autres projets plus nettement politiques, tels que le site de la « diaspora togolaise pour la démocratie », né en 1995, qui milite en faveur de « l'idéal démocratique largement partagé à travers le monde par la plupart des Togolais. Pour preuve, la contribution morale, politique et financière que la diaspora togolaise a apportée au peuple togolais durant le processus de démocratisation enclenché manifestement au début de la présente décennie $\aleph^{24}$. Il existe aussi un blog en anglais, arabe, espagnol et français de la diaspora sahraouie ${ }^{25}$, qui présente les informations politiques touchant directement ou indirectement la région du Sahara occidental et des témoignages sur la « diaspora en exil et ses souffrances ».

Contrant la connotation parfois négative du terme de diaspora dans la culture haïtienne ${ }^{26}$, l'un des sites qui la représentent, créé en 2006 à Montréa ${ }^{27}$, ville où les Haïtiens forment l'une des communautés ethniques les plus nombreuses, affirme une solidarité autour d' « une communauté unie et synthétique » et la dimension de peuple rassemblé par-delà les distances et différences. Il souhaite que « [l'on finisse] par comprendre que nous sommes un, et qu'il faut qu'on vive ensemble, ainsi bâtir un meilleur lendemain pour nous et les générations à venir puisqu'il est un fait : on est Haïtien et on le sera toujours ».

Cette politique identitaire doit être servie par un effort économique de la diaspora : « La Diaspora Haïtienne (LDH) offre aux entrepreneurs, particulièrement à ceux de la communauté, l'opportunité de promouvoir les biens et les services de leurs entreprises auprès d'une multitude de clients potentiels aussi bien d'ici qu'à travers le vaste monde $\aleph^{28}$.

La catastrophe qu'a représentée le séisme de 2010 a par ailleurs vu croître ces appels à la diaspora - «Aujourd'hui, la seule ressource qui peut être mobilisée rapidement, (...) c'est la diaspora. Je n'ai pas, moi, comme chef de gouvernement d'Haïti, d'autre alternative. (...) On a besoin de vous ", déclarait le premier ministre de l'époque ${ }^{29}$ - et l'exhortation des cadres au retour ${ }^{30}$, en même temps qu'elle provoqua un immense élan identitaire au sein de la diaspora.

Plusieurs initiatives d'orientation idéologique différente (sites officiels des États, initiatives individuelles ou associatives dans les établissements de la diaspora, sites

$23 \mathrm{http}: / /$ www.diasporas.fr/index.php

$24 \mathrm{http}: / /$ www.diastode.org/ausujet.html

$25 \mathrm{http}: / /$ diasporasaharaui.blogspot.com/

26 Si l'on en croit BÉCHACQ Dimitri (2007) La diaspora haïtienne à Paris. Significations, visibilités et appartenances, in Caraïbes Géode, Dynamiques migratoires de la Caraïbe, Paris, Karthala, pp. 253-271.

$27 \mathrm{http} / / /$ www.ladiasporahaitienne.com/

$28 \mathrm{http} / / /$ www.ladiasporahaitienne.com/Le_mot_du president fondateur.html

$29 \mathrm{http}: / /$ blogues.cyberpresse.ca/haiti/2010/01/25/la-diaspora-haitienne-interpelee/

30 Haïti lance un programme de retour pour ses cadres expatriés, Le Monde, 30 septembre 2010.

REMI 2012 (28) 1 pp. 13-31 
d'organisations humanitaires, religieuses ou politiques, etc.) se disputent ainsi l'espace virtuel des diasporas, tout en contribuant à installer leur existence dans l'espace public. L'exemple indien est ici intéressant dans la mesure où la récente introduction du terme de diaspora pour désigner les Indiens de l'extérieur correspond quasiment à la mise en place par le gouvernement de l'Inde d'une politique délibérée de valorisation des populations d'outre-mer, marquée en 2000 par la création du « Haut Conseil de la diaspora indienne » (Dumont, 2008). La diaspora possède un site officiel animé par l' « intérieur $»^{31}$ qui milite notamment pour l'obtention de la double nationalité pour les membres de la diaspora ${ }^{32}$ tandis que des initiatives sporadiques sont prises sur le web par des « extérieurs » d'horizons divers ${ }^{33}$.

Nous pouvons compléter ce modeste tour d'horizon virtuel par l'exemple d'un site anglophone à vocation humanitaire « The Diaspora Volunteering Alliance » qui réunit des associations très diverses dont l'action concerne les Sud, aussi bien en Afrique, Asie et Amérique, et revendique parfois une obédience chrétienne ou islamique. Son projet consiste à « engager et envoyer des volontaires soutenir des projets de développement dans leurs pays et continents d'origine $»^{34}$. Visant à encourager la capacité d'action des diasporas concernées (principalement les groupes de migrants installés au Royaume-Uni), ce site tend à nous indiquer le sens qu'a pris le terme de diaspora, dans le débat international à l'heure d'une « remontée des Sud » pour reprendre la belle formule d'Alain Tarrius (2007). On aurait donc ici affaire à une diaspora des Sud revendiquée, douée de capacité de mobilisation et de ressources, acteur économique et objet d'attention politique.

Tout en s'en distinguant, ces revendications ne vont pas sans faire référence aux modèles des diasporas classiques, en particulier au modèle juif avec lequel elles entretiennent des relations de mimétisme, mais aussi de distance. Nous pourrions prendre ici l'exemple de la diaspora palestinienne dont l'affirmation, après la création de l'État d'Israël et la nakba (catastrophe), a pour une large part repris les problématiques de l'exil du peuple juif. Pour prendre un autre exemple, la revendication haïtienne emprunte à l'histoire des juifs, comme le montre, dans son lyrisme, la description qui suit dans laquelle prévaut une image du pouvoir supposé de la diaspora. Préconisant la double nationalité, un blogueur déclare : «Une petite histoire comparée ne ferait de mal à personne. Prenons l'exemple le plus réaliste, celui de l'État d'Israël. Sa création, sa population, ses élites, ses dirigeants, son financement, tout ou presque, vient de la diaspora juive. Qui ne se rappelle Exodus, ces bateaux bondés sur les quais de Marseille, en partance pour la Palestine, en vue de consolider l'indépendance de l'État Hébreu. Mais, il n'y avait pas que Marseille, la diaspora juive venait de partout et aujourd'hui encore, cet État, l'un des plus puissants au monde en un peu plus d'un demi-siècle s'est constitué grâce à sa diaspora. Ce qu'on oublie toujours de dire, tous ces gens-là n'ayant jamais connu d'autres pays que leurs lieux de naissance, c'est-à-dire la France, le Canada, les États-Unis, l'Allemagne et le reste

$31 \mathrm{http}: / /$ indiandiaspora.nic.in/contents.htm

32 L'Inde considère en effet que tous les résidents extérieurs et descendants d'Indiens ayant la nationalité du pays d'installation doivent pouvoir bénéficier de la double nationalité.

33 Le réseau de la Diaspora Indienne de France, par exemple, s'adresse plutôt à des cadres et dirigeants. Consulté en décembre 2011, il affichait soixante-douze membres (http://www.viadeo. $\mathrm{com} /$ hub/accueil/?containerId=002n2z1ehfzrty6).

$34 \mathrm{http}: / /$ www.diasporavolunteeringalliance.org/about-us 
du monde n'étaient pas Israéliens au départ. Mais ils appartenaient tous, à une culture commune, le judaïsme. Les plus intransigeants aujourd'hui, sur la question de l'occupation de la Palestine, ce sont eux. Les plus nationalistes, les plus patriotes ce sont eux. Alors qu'ils ont tous ou en grande partie, la double, voire la triple nationalité. Dans ce cas, nous ne voyons pas pourquoi les Haïtiens, qui n'ont pas de leçon de patriotisme et d'amour de la patrie à recevoir, ne peuvent pas posséder une nationalité étrangère, sans jamais penser un instant à nier leur "Haïti Thomas" $\rangle^{35}$.

Pour prendre un autre exemple, la revendication corse avoue moins volontiers de liens avec le modèle du judaïsme, mais la création d'une " agence du retour » destinée à encourager les « extérieurs » à revenir sur l'île ne va pas sans évoquer le rôle de « l'agence juive » organisant le « retour» des juifs en Palestine puis en Israël ${ }^{36}$. Ainsi, comme l'indique l'un des principaux promoteurs de l'idée de « diaspora corse » interrogé par un journaliste sur ce sujet, « il n'existe pas de modèle pour la création d'une diaspora ; chaque expérience est le fruit d'une situation politique particulière et de la capacité des émigrés à s'organiser. On peut voir le cas des Palestiniens, des Juifs, des Irlandais, des Arméniens, des Basques... Bien entendu, axés sur le problème de défense des minorités, nous nous sommes intéressés en permanence à l'Histoire, très souvent tragique de ces peuples déchirés, déracinés et aux migrations qu'on leur a imposées. Nous avons nécessairement eu des réflexions induites par ces évènements, ces expériences. Notre revendication d'une "Agence du retour" puise incontestablement à ces sources communes tandis que, avec d'autres, nous avons initié "Femu Qui", société de "capital-développement", par parenté avec "Herrikoa", société basque dont le fleuron était la coopérative de Mondragon dont la visite nous avait impressionnés $»^{37}$. Au cours de ces années d'effervescence identitaire, on a pu assister ainsi à une véritable émulation entre diasporas, imitations, comparaisons, solidarités, mais aussi compétitions. Si la revendication des noirs en Europe se calque sur celle des noirs américains et si la formation du CRAN ne va pas sans évoquer le $\mathrm{CRIF}^{38}$, elle tend à distinguer des communautés et risque toujours peu prou d'encourager « l'entre-soi » (Schnapper, Bordes-Benayoun et Raphaël, 2009). Le divorce entre juifs et noirs constitue la dérive récente la plus frappante, même si elle reste minoritaire, de ces ruptures dans la solidarité quasi-naturelle qui a pu se nouer dans l'histoire entre les deux groupes (Lapierre, 2011).

Contrairement aux diasporas classiques, plutôt tournées vers l'installation dans des sociétés d'arrivée, chaque fois que celles-ci en offraient la possibilité, qui développaient des identités à trait d'union (juifs-français, sino-américains, etc.), les diasporas affichées sur le net se caractérisent par l'aspect volontaire et proclamé de leur existence

$35 \mathrm{http} / / /$ haitiensenfrance.online.fr/spip.php?article94

36 Créée en 1929 pendant la période du mandat britannique pour organiser la vie des juifs en

Palestine, l'Agence juive est aujourd'hui l'organisme israélien chargé de l'alya, c'est-à-dire la venue des juifs de la diaspora en Israël.

$37 \mathrm{http}: / /$ edmondsimeoni.blog.lemonde.fr/category/bibliographie/

38 Le Conseil Représentatif des Institutions juives de France (CRIF), né dans la clandestinité durant l'Occupation fut officiellement fondé en 1944. Le Conseil Représentatif des Associations Noires (CRAN) a été créé en 2005. À l'instar des dîners annuels du CRIF, il organise des « dîners du CRAN » où sont invités des élus pour aborder divers sujets propres à la « communauté ». L'organisation des populations noires d'Europe est un fait récent ; des associations du même type sont en cours de constitution en Autriche, en Belgique et en Italie.

REMI 2012 (28) 1 pp. 13-31 
comme collectif diasporique et de leur labellisation comme telle. En résumé, elles vantent les vertus de l'organisation et de la solidarité diasporiques, de la fierté ethnique, cèdent volontiers au mythe du pouvoir politique et économique de la diaspora comme à celui de l'unité communautaire dont elles cultivent l'idéal.

Cette nouvelle représentation sociale des diasporas dans l'espace public n'est pas sans relation avec les problématiques scientifiques. Du reste, à l'heure d'Internet, il existe une circulation des savoirs et une certaine porosité des deux domaines de connaissance. Les chercheurs sont souvent présents sur les sites diasporiques, invités au titre d'experts ou par leurs publications et le jeu des citations, quand ils n'en sont pas carrément les promoteurs. Leurs approches ont évolué elles aussi vers une mobilisation de la notion de diaspora dans des perspectives de plus en plus larges.

\section{DU TRAVAILLEUR IMMIGRÉ AU MIGRANT DIASPORIQUE}

L'entrée massive du terme de diaspora en sciences sociales correspond à un changement de perspective dans l'étude des migrations, passant progressivement de l'étude de mouvements unidirectionnels puis bipolaires à celle de mouvements transnationaux et multipolaires et de l'ethnicité locale, étudiée à l'échelle de petites entités locales et dans le cadre des États-Nations qui les accueillent et les produisent, aux identités globales transgressant les frontières nationales (Abélès, 2008). Le calendrier de l'arrivée des diasporas dans le domaine scientifique concorde avec celui évoqué plus haut à propos d'Internet. Si l'on excepte quelques écrits avant-coureurs, les années 1990 et 2000 en constituent les moments forts. Rappelons quelques publications bien connues, qui jalonnent ces années de pénétration de la thématique dans la recherche. Pour la première décennie : en 1991, création de la revue Diaspora. A Journal of Transnational Studies, publiée par l'Université de Toronto ; plusieurs publications ont marqué la décennie pour beaucoup dans les travaux anglo-saxons (Gilroy, 1993 ; Clifford, 1994 ; Cohen, 1997 notamment) et en France (Hérodote, 1989 ; Chaliand et Rageau, 1991 ; Médam, $1993^{39}$; Prévélakis, 1996). Puis les années 2000 vont consacrer la très large diffusion de la notion parmi les chercheurs en particulier en France, avec une succession de colloques et publications : Schnapper, 2001 ; création à Toulouse de la revue Diasporas, histoire et sociétés (2002); Dufoix, 2003 ; Bruneau, 2004 ; Anteby, Berthomière et Schaeffer, 2005 ; Berthomière et Chivallon 2006 ; Bordes-Benayoun et Schnapper, 2006 et 2008. Cette liste non exhaustive témoigne de la «ferveur académique » qui a porté « l'illusion de la mise en œuvre d'une pensée différente » (Chivallon, 2006). Dans un monde bouleversé dans ses cadres de référence habituels, après la chute du mur de Berlin et l'accélération des processus de globalisation, on cherche à dépasser les frontières assignées traditionnellement aux objets sociologiques et anthropologiques, que ceux-ci soient définis en termes de populations,

39 C'est également au début des années 1990 que le Centre Interdisciplinaire de Recherches et d'Études Juives du CNRS et de l'Université de Toulouse le Mirail (CIREJ) dirigé par Chantal Bordes-Benayoun, laboratoire rejoint par Alain Médam, devient dans un premier temps le CIREJED (d'études juives et des diasporas) puis quelques années plus tard le laboratoire DIASPORAS qui fondera la revue Diasporas, Histoire et Sociétés dirigée par Chantal BordesBenayoun et Patrick Cabanel. Lors du congrès de l'AISLF de 1992 à Lyon, une table-ronde « actualité des diasporas » avait été animée par les chercheurs de l'équipe. 
de groupes sociaux, d'espaces, de localités ou de cultures. Confrontés à un nécessaire renouvellement après l'essoufflement d'une période propice à la redécouverte des particularismes, les chercheurs vont alors progressivement construire leurs objets aux frontières, aux intersections et aux interactions. Au cours de ce lent déplacement de perspective, ils seront tentés de considérer toute migration, y compris quand elle est déplacement virtuel, comme une diaspora et d'y voir une condition universelle.

Trois périodes peuvent être distinguées marquées par trois figures : l'immigré, le minoritaire ethnique et l'homme de la diaspora.

\section{De l'immigré au minoritaire ethnique}

Si les années 1960 sont celles de la mesure des flux et de la figure du « travailleur immigré » principalement considéré comme force de travail, la redécouverte des cultures au sein des sociétés nationales occidentales postcoloniales occupera les recherches jusqu'à la fin des années 1980 qui s'organiseront autour de l'objet " ethnique ». Puis, l'arrivée de «l'homme de la diaspora » (Bordes-Benayoun et Schnapper, 2006), mais aussi du « nomade », du transnational ou du cosmopolite, marquera à son tour l'affaissement de la fièvre ethnique qui s'était emparée de la recherche. Ce sont autant de figures de l'étranger, sans doute la seule condition universelle plausible, qui organisent les recherches.

Quand elle n'était pas totalement absorbée comme une composante surexploitée de la classe ouvrière, la figure de l'immigré fut donc souvent réduite à sa dimension d'utilité économique et envisagée à partir de la seule société dite d'accueil. Dans le contexte des années 1960, où dominait le marxisme dans ce champ de la recherche, les immigrés représentaient « une force de travail temporaire, d'hommes seuls, en marge du système social » (Rea et Tripier, 2003 : 25-26). Cette victimisation se poursuivit assez longtemps avant que l'on ne bascule au contraire vers une vision parfois excessive de la migration comme aventure subjective.

Mais avant cela, l'étape de l'affirmation ethnique occupa les débats publics et universitaires. Depuis l'irruption en France et outre atlantique du thème de la différence, dans un contexte postcolonial de critique du modèle d'assimilation, c'est principalement autour de la figure du « minoritaire ethnique » qu'allaient s'orienter les recherches. La liste des travaux qui s'engagèrent des années 1970 au début des années 1990 serait bien trop longue pour être dressée ici. Il s'agissait de pallier les effets d'occultation que la politique de l'intégration dite « à la française » ici (Noiriel, 1988) et celle du melting pot là-bas (Glazer et Moynihan, 1970) avaient produits jusque dans la recherche et prendre réellement en considération les différenciations culturelles trop longtemps ignorées ou minimisées. Il est du reste intéressant de noter comment, à la fin des années 1970, Glazer analyse le succès de son livre, qui allait devenir un classique de la sociologie et dont la première édition remonte au début des années 1960 : «The book's impact, I believe, was based on the fact that it took ethnicity seriously at a time when most thinking people considered it a survival from the age of mass immigration and of decreasing importance. It turned out we - in America and the rest of the world-were due for a revival of ethnic interests, 
loyalties, and attachments in the later 1960s and 1970s, accompanied by considerable social research on ethnicity $\gg^{40}$.

L'importance de l'ethnique dans les recherches n'eut d'égale que la diffusion sociale du terme. Ainsi, dans les enquêtes que j'avais pu conduire pour ma part sur la « minorité juive », au nom d'une réhabilitation de la différence culturelle, si valorisée dans le contexte des années 1980, certaines personnes interrogées, souvent héritières du francojudaïsme hier dénommées « israélites », et s'affirmant désormais « juives », s'amusaient de se voir désignées comme « ethniques »! C'est que l'ethnique commençait à devenir un paradigme dominant dans la recherche et une mode dans la vie quotidienne (Raulin, 2000 : 10). Les études sur les identités et les minorités se multipliaient. Le terme de diaspora en était absent ou réservé aux diasporas classiques. Mais même appliqué aux juifs, son emploi s'inscrivait dans l'air du temps. Le livre de Richard Marienstras, que l'on doit à juste titre considérer comme pionnier et visionnaire pour le développement d'une socio-anthropologie des diasporas ${ }^{41}$, n'en adoptait pas moins le point de vue ambiant et antijacobin de défense des minorités et des cultures contre leur nivellement. « Dispersés, désunis, les minoritaires ne sont rien. Unis, ils pourront graduer et organiser la lutte contre l'Étatnation, revendiquer une part de souveraineté (...)» (Marienstras, 1975 : 98). Les Juifs de la diaspora avaient une "vocation minoritaire » et devaient organiser leur existence propre au sein des sociétés d'accueil au même titre que «les Tziganes, ou les Catalans, les Basques, le Bretons, le Indiens, les Slovènes, (...) les Arméniens » (ibid. : 61). Reflet d'un engagement et d'une époque, le livre avait signalé déjà les effets réducteurs sur le plan heuristique de la victimisation de la diaspora, souvent vue comme une condition douloureuse et annihilante. Marienstras invitait au contraire à ne pas la considérer sous le seul effet de la domination, mais dans toute sa capacité créatrice. Cette perspective novatrice ne sera développée que bien plus tard dans les sciences sociales (notamment Boyarin et Boyarin, 2002). On doit aussi à Marienstras une belle formule pour définir la diaspora, dont « le centre est partout et la circonférence nulle part ».

L'article de John A. Armstrong (1976), paru à la même époque, marque également ces débuts précoces d'une problématique et d'une typologie des diasporas. Toutefois, sa réflexion ne s'en inscrivait pas moins dans celle relative à l'ethnicisation des rapports sociaux. À cette époque, la notion de diaspora ne se démarquait guère de celle de groupe ethnique.

Malgré toutes les précautions que l'on pouvait prendre pour déjouer les risques d'essentialisation que portait en germes le concept d'ethnicité, il dérivait bel et bien du terme « ethnie » dont Colette Guillaumin dans un brillant ouvrage n'avait pas manqué de signaler les ambiguïtés tant il restait proche du terme de " race ». Il témoignait d'une « organisation mentale inconsciente de la saisie de l'autre » dont il semblait particulièrement difficile de s'affranchir. "L'adoption d'un concept comme celui "d'ethnie" a donc été une tentative de distance envers les connotations héréditaires et constitutionnalistes qui

40 Article de Nathan Glazer, http://www.garfield.library.upenn.edu/classics1980/ A1980HX94000001.pdf

41 Un extrait du livre de Marienstras a été republié avec l'accord de son auteur, hélas disparu récemment, dans le numéro 2 de Diasporas, histoire et sociétés, dans la rubrique « Classiques de l'histoire et de la sociologie des diasporas » (2003). 
marquaient le terme race. Mais ce fut une vaine tentative, puisque l'emploi courant de ce mot, que le vocabulaire de la recherche a fait passer dans le domaine général, a récupéré ce sens qui prend décidément figure inéliminable »(Guillaumin, 1972 : 60-61). Certes l'ethnicité des sciences sociales n'est pas équivalente à l'ethnie des premiers temps de l'ethnologie, mais l'insistance avec laquelle les chercheurs entendaient se démarquer de toute démarche essentialiste, en particulier des théories primordialistes, est à la mesure des difficultés inhérentes à leurs catégorisations de l'étranger, dont seule la posture d'entredeux décrite pas Simmel puisse en définitive rendre compte sans la réduire ni l'enfermer (Simmel, 1908). Dans le même sens, on ne peut que se féliciter de voir revisitée la figure de « l'homme marginal » (Cuche, 2009). Répétée à l'envi, la mise à distance vis-à-vis du communautarisme sous-jacent à l'affirmation ethnique, ne parvenait pas pour autant à venir à bout de cette étrange alchimie entre l'intériorité et l'extériorité, la fidélité aux siens et l'entrée en relation avec d'autres, la participation sociale ici et maintenant et la mémoire des origines, qui constitue l'univers de l'étranger. À son corps défendant l'approche de «l'ethnique » renvoyait inlassablement ce dernier vers ses origines idéalisées dans la perspective d'une réparation des méfaits des politiques d'assimilation et des échecs de l'intégration. Elle privilégiait toujours plus ou moins l'extériorité culturelle et géographique au détriment de l'universel et de l'aspiration universaliste des hommes et des femmes ainsi caractérisés et qui étaient souvent désireux de sortir de la marginalité où ils avaient été rejetés. Elle insistait enfin sur la fragmentation sociale résultant de la coexistence difficile de groupes d'abord définis par leurs cultures et la nécessité de les reconnaître dans leurs particularismes dont rendraient compte théories et politiques multiculturalistes ${ }^{42}$.

La critique de l'assimilation conçue comme une inculcation imposée ne laissait guère de place à la prise en compte de la volonté d'intégration des migrants dans certaines conditions. L'intégration (républicaine) devint une sorte de mot tabou dans la recherche non sans quelque confusion entre l'action intégratrice de la société dans son ensemble, au sens durkheimien, qui concerne non pas tel ou tel individu, mais la totalité d'une organisation sociale et une intégration faisant l'objet de décret (Schnapper, 2007). La «politique d'intégration » décrétée entend intégrer volontairement un groupe particulier, et a pour effet, qu'on le veuille ou non, de désigner des «non-intégrés », c'est-à-dire de conforter les processus d'assignation ethnique ou sociale dont l'efficacité sociale n'est pas négligeable. Cette confusion entre le processus et la volonté a souvent conduit à déplacer sans vraiment la quitter la problématique de la domination.

L'ethnicité, « nouveau concept, (dont) la portée (dépassait) largement l'objet ethnique pour alimenter une révision des paradigmes antérieurs » (Poutignat et StreiffFeinart, 1995 : 31) n'allait pas forcément tenir ses promesses. Malgré toutes les précautions pour dynamiser leurs approches dans une perspective relationnelle ou interactionniste, les chercheurs furent confrontés d'un côté, à la réalité grandissante des replis ethniques et communautaires, qui interrogeaient le risque de clôture de leurs catégorisations ; de l'autre, à l'expression plus individuelle, multiple et polymorphe, d'identités sortant, bien au-delà de toutes prévisions, des frontières des groupes considérés et sortant du simple jeu de miroir que l'interactionnisme d'un Fredrik Barth avait invité à considérer.

42 Dans leurs évolutions, les travaux de Didier Lapeyronnie, Michel Wieviorka ou de Marco Martiniello rendent comptent à leur manière de ces débats intérieurs autour de la fragmentation, des limites de l'ethnicité et de la nécessité de « sortir des ghettos culturels ».

REMI 2012 (28) 1 pp. 13-31 
Plus avant le paysage des migrations ayant changé à la fois dans son ampleur et dans ses directions, la nécessité de saisir l'importance des échanges et mouvements multipolaires de la mondialisation s'imposa de plus en plus.

\section{De l'ethnique au diasporique}

La problématique des diasporas intervient dans ce contexte et tente d'opérer une rupture par rapport aux travaux sur l'ethnicité et les identités culturelles. Les sciences sociales inscrivent résolument alors le mouvement dans leurs approches de l'étranger. Celui-ci doit dès lors être envisagé dans tous ses déplacements. Spécialiste de l'immigration, Abdelmalek Sayad avait alerté les sociologues dès 1991 sur l'expérience duelle de la migration, entre présence et absence, entre une terre de départ que l'on ne quitte jamais totalement et une terre d'arrivée où l'on s'installe parfois plus longtemps que prévu. Ce qui peut être écrit aujourd'hui à propos de l'exil et du retour de l'homme de la diaspora n'a rien à envier à la force de certaines de ses évocations. La vision de l'immigré repose, écrivait-il, « sur l'illusion collective d'un état qui n'est ni provisoire ni permanent » (Sayad, 1991 : 149). Sans doute était-ce là l'amorce d'un tournant dans l'étude des migrations qui néanmoins ne quittait pas encore totalement les théories de la domination qui faisaient de « l'étranger » l'objet d'une particularisation dans un rapport inégal.

L'étude des diasporas fut donc censée résoudre la difficulté éprouvée par les chercheurs à rendre compte, contre la tentation de réification des identités ethniques, de leur fluidité, de leur variabilité voire de leurs caprices dans un monde où frontières et nations semblaient vaciller sous l'effet de la complexification des échanges et des flux. Pari difficile, illusoire peut-être, que ce choix du mouvement et de la fluidité que manifeste l'arrivée de la notion de diaspora. L'utilité du concept tient à l'approche de l'espace qu'elle permet. Selon une acception classique, on peut définir la diaspora comme la dispersion géographique d'un peuple qui conserve le sentiment d'une unité en dépit de la distance et cultive la volonté de se référer à une histoire commune, nourrit un mythe du retour consigné dans un récit élaboré et transmis de génération en génération. La dimension territoriale y est à la fois importante, mais toujours liée à l'histoire et à la mémoire des dispersés.

Le mérite premier de la problématique des diasporas est certainement d'avoir replacé les migrants - terme qui a définitivement remplacé celui d'immigré et ne préjuge donc pas de la direction des parcours - dans un vaste espace, plus large que celui reliant pays de départ et pays d'arrivée. Ce n'est pas seulement parce que la réalité migratoire et géopolitique contemporaine a changé d'échelle que cette perspective spatiale s'est imposée, c'est aussi parce que l'attention plus grande portée aux parcours individuels et à la subjectivité de celles et ceux qui les accomplissent impliquait de considérer avec plus de profondeur le rapport aux espaces parcourus et le sens que chaque lieu, chaque territoire traversé pouvaient revêtir. Le modèle de la diaspora s'impose alors comme celui qui donne à voir des relations réelles et symboliques entre des territoires multiples qui finissent par s'imposer au fil du temps comme autant de centralités de la diaspora. Loin de la relation binaire résumant les migrations traditionnelles, l'expérience spatiale multipolaire de la diaspora peut se décrire comme une relation triangulaire entre "ici, là-bas, ailleurs », qui sont autant d'espaces d'identification, mais aussi de relations concrètes réelles ou 
possibles (Bordes-Benayoun, 2002). Ici, dans un territoire d'installation, de passage ou de circulation (Tarrius, 2007), là-bas le pays de départ(s) et de retour(s), ailleurs (les territoires de destination possible où d'autres de la famille ou du pays sont installés). Ainsi la notion essaie de tenir ensemble les réalités concrètes, mais aussi les horizons symboliques dans et vers lesquels les hommes évoluent. C'est une gageure, que relève Emmanuel Ma Mung : « la valeur heuristique (du terme de diaspora) demeure parce que l'expérience particulière qu'il s'apprête à désigner ne cesse de mettre en relation ce qu'il est d'usage de séparer, dans cette tension entre le paradigme du lieu (union, contiguïté) et celui de l'éloignement (séparation, dispersion) » (Ma Mung, 2006).

Mais l'usage du terme répond-il toujours à cette exigence ? Son extension à l'infini, constatée par nombre de chercheurs, n'affaiblit-elle pas sa pertinence sociologique ? Il faut tout d'abord remarquer l'ambiguïté du terme quand il désigne tour à tour un phénomène (la dispersion géographique d'un peuple), une population dispersée (la diaspora juive, la diaspora arménienne, la diaspora noire) ou un segment local d'une population dispersée (la diaspora chinoise de New York). À en juger par les contributions réunies dans des revues ou actes de colloques sur le thème, cette dernière acception est sans doute la plus fréquente dans les recherches tournées vers l'analyse de « diasporas » localisées, qui continuent de fait à être abordées comme des groupes ethniques. La saisie des relations intra-diasporiques n'est pas facile à réaliser sur le plan méthodologique et ce malgré le développement de l'anthropologie multi-sites par exemple. On est encore loin de l'ethnographie cosmopolite préconisée par Appadurai (1996 : 92-93).

Mais plus avant, à l'instar de ce que nous avons pu observer à travers quelques usages sociaux du terme diaspora sur le net, l'usage sociologique n'est pas à l'abri d'une vision communautaire, et peut aussi sacrifier au mythe de l'unité diasporique. La valorisation de la diaspora s'explique pour partie par la fascination qu'exercent la solidarité et le sens de l'entraide, stéréotypes qui sont volontiers associés aux diasporas, face aux excès de l'individualisme. Cette surestimation du communautaire conduit alors à mésestimer la dispersion. Or le phénomène de la diaspora, au sens de la dispersion, est exponentiel. La dispersion affecte le groupe lui-même, non seulement pour des raisons compréhensibles, liées à la diversification linguistique et culturelle engendrée par l'éclatement spatial, mais aussi parce que les relations aux autres sont constamment retravaillées dans l'expérience diasporique. Chaque segment de la diaspora joue donc une partition certes commune, mais aussi dissociée de l'ensemble. Cette atomisation est sensible dans la diversité des formes d'organisation concrète de la diaspora, leur « cacophonie » interne et la variété de ses registres religieux ou culturels, de ses interprétations parfois contradictoires du récit commun sans compter les écarts que les choix et les trajectoires individuelles creusent en se distinguant du destin collectif. Le monde juif ici nous montre ce caractère kaléidoscopique de la diaspora qui assemble tant bien que mal, sans qu'ils ne se ressemblent, un hassid new yorkais, un bijoutier d'un souk marocain, une bourgeoise israélite parisienne et un juif athée. L'hétérogénéité des représentations des diasporas qui ressort de l'observation de l'espace virtuel, alors que cet espace n'est pourtant pas accessible à tous leurs membres, donne un petit aperçu de cette tautologique « dispersion de la diaspora ». L'évolution des membres de la diaspora au cours de l'histoire, leurs parcours singuliers peuvent aussi aboutir à leur sortie de la « communauté » diasporique alors que tout semble 
concourir, sur le net, dans les médias, dans les politiques identitaires et dans les productions savantes à en produire et en confirmer l'existence.

Parler de diaspora pour toutes les migrations ne facilite pas non plus la précision attendue du concept. Or, comme le souligne très justement Jacques Barou, « toutes les migrations n'aboutissent pas à la constitution de diasporas. Beaucoup s'assimilent au fil du temps à la société dans laquelle elles se sont installées et perdent toute référence à leur pays d'origine. Certaines, sans parvenir véritablement à trouver leur place dans leur pays de résidence et sans perdre tout contact avec leur pays d'origine, ne sont cependant pas dans une position sociale et économique qui leur permettrait d'activer les liens avec leur zone de provenance. Leur identité se recroqueville peu à peu autour de la petite communauté très localisée qu'elles ont reconstituée dans leur pays d'accueil sans pouvoir s'élargir par la fréquentation de groupes semblables à eux et dispersés sur une plus vaste échelle » (Barou, 2007 : 151). Pour être pertinente, l'importance accordée aux initiatives des migrants au cours des dernières décennies qui a aussi nourri la thématique des diasporas dans la recherche n'en comporte pas moins le risque de surestimer leur liberté d'action et d'oublier le nœud de contraintes et d'obstacles que la plupart doivent affronter de manière parfois ô combien tragique. Ne pas victimiser n'implique pas pour autant l'absence de victime et de souffrance ! Sans doute la notion de " diasporas prolétaires » d'Armstrong a-t-elle ouvert la voie vers la réalité, si prégnante aujourd'hui quand les frontières se ferment, des migrants dépourvus de ressources à mobiliser, isolés dans leurs itinérances et livrés au bon vouloir ou à l'arbitraire des États et dont les parcours témoignent néanmoins d'une ingéniosité pour tenter de se déplacer. Peut-on employer le même terme pour toutes ces formes de migration et de transmigration que le monde contemporain nous donne à voir? Ne faut-il pas trouver d'autres termes ou enrichir le vocabulaire des diasporas d'épithètes qui pourraient en rendre compte avec plus de précision?

Sans doute ne sommes-nous pas encore finalement sortis de l'obsession ethnique. C'est souvent par le prisme communautaire que passe notre regard sur des expériences de plus en plus nombreuses identifiées comme diasporas.

L'extension du terme à des expériences, sans référence territoriale aucune, comme le font les auteurs des Postcolonial Studies, qu'accompagne un refus du point de vue de l'authenticité ethnique, n'en accorde pas moins d'importance à ce qui réunit le groupe diasporique, en somme un lien communautaire imaginaire, non fondé sur une référence historique unique. Ce qui fait dès lors la diaspora, c'est avant tout le caractère multiple des références, l'hybridité et le mélange de références parfois contradictoires et constamment changeantes. "The diaspora experience as I intend it here is defined, not by essence or purity, but by the recognition of a necessary heterogeneity and diversity; by a conception of "identity" which lives with and through, not despite, difference; by hybridity. Diaspora identities are those which are constantly producing and reproducing themselves a new, through transformation and difference » (Hall, 1994).

L'extrapolation du terme de diaspora aux identités sexuelles a parachevé cette rupture de la définition avec la définition classique qui, elle, même quand la terre d'origine n'est plus qu'une évocation lointaine et mythique, constitue la dimension de dispersion spatiale comme condition première. Dans cette vision anhistorique, la diaspora « devient 
une représentation, un discours, une revendication » (Helly, 2006 : 14). L'invention d'une Queer diaspora illustre parfaitement cette communauté proclamée dont le net en première partie de cet article donnait un aperçu. Mouvement activiste qui conteste la norme hétérosexuelle occidentale, le mouvement queer qui s'est développé depuis le début des années 1990 est représenté dans les Gender Studies et dans les études sur les migrations. La recherche met l'accent sur la variabilité et la mobilité des frontières de genre pour justifier la constitution d'une diaspora dont la formation serait encouragée par la mondialisation des échanges et la circulation. " "Queer Diaspora" intervenes at the intersection of queer studies and Diasporas studies. It explores how the conditions of geographical mobility produce new experiences and understandings of sexuality and gender identity on the one hand, and how the (hetero-) normative and queer articulations of subjectivity emerge in those trans-national and cross-cultural movements on the other hand. As we are "queering" diasporas it is important to examine the critical slippage between queer and diasporas, the new articulations of normative and subversive subjectivity emerging from the material, geographical and ideological displacements of globalization $»^{43}$.

Plus généralement, l'extension à l'infini de la notion de diaspora telle qu'elle est mise en œuvre dans cet exemple, manifeste, à travers l'objectif subversif annoncé et l'activisme des chercheurs et militants, le caractère éminemment politique qu'a pu prendre dans les derniers temps la notion de diaspora. Alors que le mythe de l'unité communautaire continue de nourrir l'engouement des chercheurs pour le nouveau concept, son extension met l'accent sur la mobilité tous azimuts que la globalisation est censée développer. Une fascination pour le déplacement que ne partagent pas forcément les déplacés...

43 Appel à communication pour le First International Conference on Queer Diaspora, organisé par le Département des gender studies de l'Université de Taïwan, en 2011. 


\section{Références bibliographiques}

ABÉLÈS Marc (2008) Anthropologie de la globalisation, Paris, Payot, 280 p.

ANTEBY-YEMENI Lisa, BERTHOMIÈRE William et SHEFFER Gabriel (Dir.) (2005) Les diasporas. 2000 ans d'histoire, Rennes, Presses Universitaires de Rennes, 497 p.

APPADURAI Arjun (2005 [1996]) Après le colonialisme. Les conséquences culturelles de la globalisation, Paris, Petite Bibliothèque Payot, $331 \mathrm{p}$.

ARMSTRONG John A. (1976) Mobilized and Proletarian Diasporas, American Political Science Review, 70 (2), pp. 393-408.

BAROU Jacques (2007) La planète des migrants, Presses Universitaires de Grenoble, $180 \mathrm{p}$.

BERTHOMIÈRE William et CHIVALLON Christine (Dir.) (2006) Les diasporas dans le monde contemporain, Paris, Karthala - MSH Aquitaine, 415 p.

BORDES-BENAYOUN Chantal (2002) Revisiter les diasporas, Diasporas, histoire et sociétés, 1, pp. 9-21.

BORDES-BENAYOUN Chantal (2008) Les diasporas ou l'expérience de l'unité dans la diversité, Hermès, 51, pp. 189-193.

BORDES-BENAYOUN Chantal et SCHNAPPER Dominique (2006) Diasporas et nations, Paris, Odile Jacob, 255 p.

BORDES-BENAYOUN Chantal et SCHNAPPER Dominique (2008) Les mots des diasporas, Toulouse, Presses Universitaires du Mirail, $127 \mathrm{p}$.

BOYARIN Daniel et BOYARIN Jonathan (2009 [2002]) Pouvoirs de diaspora, Paris Éditions du Cerf, $167 \mathrm{p}$.

BRUNEAU Michel (2004) Diasporas et espaces transnationaux, Paris, Anthropos, 249 p.

CHALIAND Georges et RAGEAU Jean-Pierre (1991) Atlas des diasporas, Paris, Odile Jacob, $183 \mathrm{p}$.

CHIVALLON Christine (2006) Introduction. Diaspora : ferveur académique autour d'un mot, in William Berthomière et Christine Chivallon Dir., Les diasporas dans le monde contemporain, Paris, Karthala - MSH Aquitaine, p. 18.

CLIFFORD James (1994) Diasporas, Cultural Anthropology, 9 (3), pp. 302-338.

COHEN Robin (1997) Global Diasporas. An introduction, Londres, Routledge, 228 p.

CUCHE Denys (2009) «L'homme marginal » : une tradition conceptuelle à revisiter pour penser l'individu en diaspora, Revue Européenne des Migrations Internationales, 25 (3), pp. 13-30.

DUFOIX Stéphane (2003) Les diasporas, Paris, Presses Universitaires de France, 127 p.

DUMONT Gérard-François (2008) Un nouvel acteur géopolitique : la diaspora indienne, Géostratégiques, 19, [en ligne]. URL : http://www.strategicsinternational.com/19_03.pdf

FAUVELLE-AYMARD François-Xavier (2006) Les diasporas africaines, Internet et la formation d'une nation noire intercontinentale, in William Berthomière et Christine Chivallon Dir., Les diasporas dans le monde contemporain, Paris, Karthala - MSH Aquitaine, pp. 175-185.

GILROY Paul (1993) The black Atlantic. Modernity and Double Conciousness, Londres, Verso, 252 p.

GLAZER Nathan and MOYNIHAN D. Patrick (1970) Beyond the melting pot: the Negroes, Puerto Ricans, Jews, Italians, and Irish of New York City, Cambridge, MA - MIT, 363 p.

GUILLAUMIN Colette (1972) L'idéologie raciste. Genèse et langage actuel, Paris, Éditions Mouton, $247 \mathrm{p}$.

HELLY Denise (2006) Diaspora : un enjeu politique, un symbole, un concept?, Espace, Populations, Sociétés, 1, pp. 17-31.

LACOSTE Yves (Éd.) (1989) Géopolitique des diasporas, Hérodote, 53, 209 p.

LAPIERRE Nicole (2011) Causes communes. Des Juifs et des Noirs, Paris, Stock, 328 p.

LE BAYON Simon (2010) Sociologie de la composition des collectifs web 2.0. Le cas de la diaspora bretonne, Thèse de Sociologie, Université de Haute-Bretagne Rennes 2, [en ligne]. URL : https://docs.google.com/leaf?id=0Bw_mnqSYpuJOMzQ3MmQ4OGItNzAzMy00YzJiLTgzZjc tNTNIN2FmZDgyZjlk\&sort=name\&layout=list\&num=50 
MARIENSTRAS Richard (1975) Etre un peuple en diaspora, Paris, François Maspero, 213 p.

MATTELART Tristan (2009) Les diasporas à l'heure des technologies de l'information et de la communication : petit état des savoirs, TIC et Société, 3 (1), pp. 11-57.

MA MUNG Emmanuel (2006) Conclusion générale. Les vêtements neufs de la diaspora. Digressions sur les paradoxes diasporiques, in William Berthomière et Christine Chivallon Dir., Les diasporas dans le monde contemporain, Paris, Karthala - MSH Aquitaine, pp. 409-415.

MÉDAM Alain (1993), Diaspora/diasporas. Archétype et typologie, Revue Européenne des Migrations Internationales, 9 (1), pp. 59-66.

NOIRIEL Gérard (1988) Le Creuset Français. Histoire de l'immigration. XIXe-XXe siècles, Paris, Seuil, $441 \mathrm{p}$.

NEDELCU Mihaela (2009) Du brain drain à l'e-diaspora : vers une nouvelle culture du lien à l'ère du numérique, TIC et Société, 3 (1), pp. 152-173.

PRÉVÉLAKIS Georges (Dir.) (1996) Les réseaux des diasporas, Paris, L’Harmattan, 444 p.

PROULX Serge, POISSANT Louise et SÉNÉCAL Michel (Dir.) (2006) Communautés virtuelles. Penser et agir en réseau, Québec, Presses de l'Université Laval, 361 p.

RAULIN Anne (2000) L'ethnique est quotidien. Diasporas, marchés et cultures métropolitaines, Paris, L'Harmattan, $225 \mathrm{p}$.

REA Andrea et TRIPIER Maryse (2003) Sociologie de l'immigration, Paris, La Découverte, 120 p.

SCHNAPPER Dominique (2001) De l'État-nation au monde transnational. Du sens et de l'utilité du concept de diaspora, Revue Européenne des Migrations Internationales, 17 (2), pp. 9-36.

SCHNAPPER Dominique (2007) Qu'est-ce que l'intégration ?, Paris, Gallimard/folio, 238 p.

SCHNAPPER Dominique, BORDES-BENAYOUN Chantal et RAPHAËL Freddy, La condition juive en France. La tentation de l'entre-soi, Presses Universitaires de France, 2009, 152 p.

TARRIUS Alain (2007) La remontée des Sud. Afghans et Marocains en Europe méridionale, Paris, Éditions de l'Aube, $202 \mathrm{p}$ 


\title{
La diaspora ou l'ethnique en mouvement
}

\author{
Chantal Bordes-Benayoun
}

Que révèlent les usages de plus en plus fréquents du terme diaspora dans la vie sociale et dans la vie scientifique? Si la diaspora a pris une valeur positive aujourd'hui c'est que lui sont prêtées des vertus économiques et politiques qui tranchent avec la vision classique des diasporas marquées par l'exclusion et le rejet. La multiplication des sites consacrés aux diasporas depuis le début des années 2000, qui forment un ensemble disparate, donne la mesure de l'installation et de la banalisation des diasporas dans l'espace public. L'arrivée du terme dans les sciences sociales à partir des années 1990 marque l'épuisement relatif des problématiques en termes d'immigration puis d'ethnicité. Si la recherche est plus attentive au mouvement et aux interactions, le mythe de l'unité communautaire continue de nourrir l'engouement des chercheurs pour le nouveau concept.

\section{Diaspora: A Moving Ethnicity}

\section{Chantal Bordes-Benayoun}

What does the more and more frequent use of the word "Diaspora" in the public and the academic sphere mean? Diasporas have gone up in value because they are supposed to hold economic and political virtues contrasting sharply with the classical view of Diasporas which is characterized by exclusion and rejection. The proliferation of websites devoted to Diasporas since the early 2000s has shown in different ways the spread of the notion and its settlement and trivialization in the public sphere. The word emergence in the social sciences from the 1990s reveals the limits of the approaches in terms of immigration and secondly of ethnicity. If the social scientists are today more attentive to the movement and interaction, it doesn't mean they are less fascinated by the myth of the communitarian unit that goes on nourishing their enthusiasm for the new concept.

\section{La diáspora o lo étnico en movimiento}

\section{Chantal Bordes-Benayoun}

¿Que revelan los usos cada vez más frecuentes del término diáspora en la vida social y en la vida científica? Si la diáspora ha tomado un valor positivo hoy en día, es porque se le atribuyen virtudes económicas y políticas que contrastan con la visión clásica de las diásporas determinada por la exclusión y el rechazo. La proliferación de sitios Internet dedicados a la diáspora desde comienzos del siglo XXI, que forman un conjunto heteróclito, es un indicador de la instalación y de la banalización de las diásporas en el espacio público. La utilización de ésta término en ciencias sociales desde 1990 marca la disminución relativa de las problemáticas en términos de inmigración y de etnicidad. Si actualmente la investigación se interesa ante todo a la circulación y a las interacciones, el mito de la unidad comunitaria continúa a acrecentar el entusiasmo de los investigadores por el nuevo concepto. 